Revue internationale P.M.E.

Économie et gestion de la petite et moyenne entreprise

\title{
Les chances de succès des petites entreprises : vers un diagnostic d'émergence
}

\section{Alain Capiez}

Volume 5, numéro 2, 1992

URI : https://id.erudit.org/iderudit/1008141ar

DOI : https://doi.org/10.7202/1008141ar

Aller au sommaire du numéro

Éditeur(s)

Presses de l’Université du Québec

ISSN

0776-5436 (imprimé)

1918-9699 (numérique)

Découvrir la revue

Citer cet article

Capiez, A. (1992). Les chances de succès des petites entreprises : vers un diagnostic d'émergence. Revue internationale P.M.E., 5(2), 103-132. https://doi.org/10.7202/1008141ar
Résumé de l'article

$\mathrm{n}$ a beaucoup écrit sur le risque de défaillance des entreprises, mais beaucoup moins sur les chances de succès des petites entreprises. Le but de ce travail est de proposer un diagnostic d'émergence des petites et très petites entreprises fondé essentiellement sur des variables qualitatives relatives au comportement du dirigeant et à l'organisation de l'entreprise, en utilisant les techniques statistiques d'analyse factorielle. Cette méthode de diagnostic, testée sur une population limitée, permet une détection d'entreprises à possibilités réelles d'émergence, à partir de bases solides. 


\title{
Note de recherche
}

\section{Les chances de succès des petites entreprises: vers un diagnostic d'émergence}

Alain CAPIEZ*

Université d'Angers

\section{RÉSUMÉ}

On a beaucoup écrit sur le risque de défaillance des entreprises, mais beaucoup moins sur les chances de succès des petites entreprises. Le but de ce travail est de proposer un diagnostic d'émergence des petites et très petites entreprises fondé essentiellement sur des variables qualitatives relatives au comportement du dirigeant et à l'organisation de l'entreprise, en utilisant les techniques statistiques d'analyse factorielle. Cette méthode de diagnostic, testée sur une population limitée, permet une détection d'entreprises à possibilités réelles d'émergence, à partir de bases solides.

\begin{abstract}
There is an abundant literature about the risk of company failure, but few things are written about the prediction of small business success. The purpose of this research is to present a diagnosis of smaller business rising; this diagnosis, founded on qualitative variables such as the manager's behaviour and the firm's organisation, uses factor analysis statistical technics. The method we present, tested with a small population, is an efficient way to detect firms with genuine facilities of rising out.
\end{abstract}

* Alain Capiez est enseignant à l'Université d'Angers et consacre ses recherches à la finance d'entreprises et aux stratégies de développement des petites entreprises. Auteur d'un manuel de gestion financière, il a publié de nombreux articles sur le crédit-bail, les problèmes financiers des firmes et sur l'émergence des petites entreprises. Adresse : Faculté de Droit, d'Économie et de Sciences sociales, 11, boulevard Lavoisier, 49045 Angers, Cedex 01, France. 


\section{RESUMEN}

Se ha escrito mucho sobre el riesgo de quiebra de la empresas, pero poco sobre las posibilidades de éxito de las pequeñas empresas. El objectivo de este estudio es de proponer un diagnóstico de emergencia para las empresas pequeñas y también muy pequeñas. Este diagnóstico, fundado en variables cualitativas sobre el compartamiento del dirigente y sobre la organizacion de la empresa, utiliza el método estadístico del análisis factorial. Este procedimiento de diagnóstico, sometido a un test sobre una población limitada, permite seleccionar empresas que puedan desarrollarse con reales posibilidades.

\section{Introduction}

On a beaucoup écrit sur le risque de défaillance des entreprises, en raison du nombre considérable de cessations d'activité depuis le milieu des années 70 , mais la littérature reste limitée sur les chances de succès des petites entreprises (PE) et des très petites entreprises (TPE). Pourtant, de plus, depuis cinq ans, les plans-emplois du gouvernement privilégient le soutien aux PME-PMI en France ; elles représentent un potentiel important de création d'emplois, à une époque où les grandes entreprises, qui n'en finissent pas de se restructurer, congédient plus qu'elles n'embauchent.

Comment sélectionner des PE et TPE susceptibles d'émerger ? C'est à cette question que nous tenterons d'apporter une réponse. Dans une étude précédente (Capiez, 1988), nous avons défini l'émergence comme « l'obtention d'une masse critique nécessaire pour réaliser la survie et la pérennité de la TPE, en affirmant sa compétitivité dans le ou les domaines d'activité stratégique (DAS) visés ». Il convient de commenter cette définition. D'après les résultats de l'étude citée ci-dessus, l'émergence s'effectue en deux stades : un stade de démarrage-montée en compétitivité et un stade de réussite-croisière ou de réussite-développement. Ces deux stades peuvent être comparés aux stades d'existence et de maintien, puis de réussite et d'essor chez Churchill et Lewis (1983), ainsi qu'aux stades de démarrage et de montée en compétitivité, puis de régime de croisière et de développement chez Marchesnay (1986). L'émergence apparaît ainsi comme un processus dynamique de montée en compétitivité dont il faut rechercher les éléments déclencheurs. La notion de masse critique à laquelle nous nous référions implique un facteur productif et un facteur commercial ; l'étude citée met en évidence des bases internes et des bases externes de compétitivité ; les bases internes sont le dirigeant et sa capacité stratégique ; les bases externes s'expriment dans la relation de l'entreprise au marché : il s'agit de la capacité productive (technologie et organisation de la production) et de la recherche constante de débouchés face à une concurrence active. Les déclen- 
cheurs de l'émergence nous paraissent être un ou plusieurs DAS, des biens et services appropriés, des capacités productives adaptées reposant sur une technologie adéquate, et un dirigeant possédant une réelle capacité stratégique.

La sélection des PE et TPE susceptibles d'émerger demande un diagnostic d'émergence. Pour cela, nous pouvons nous inspirer des études et réflexions déjà menées sur le problème inverse, la prévision de défaillance, ainsi que celles consacrées au développement des petites entreprises. Nous en déduirons les éléments d'un diagnostic d'émergence que nous testerons sur un échantillon de PE et TPE.

\section{Causes de faillite et diagnostic de vulnérabilité}

L'analyse des causes de défaillance porte à la fois sur des éléments internes et externes à l'entreprise; elle débouche sur un diagnostic de rentabilité.

\subsection{Causes internes et externes de vulnérabilité des entreprises}

Les causes internes de faillite sont l'incompétence des dirigeants et un mauvais système de décision ; les causes externes résident dans l'incapacité de l'entreprise à faire face à la concurrence.

\subsubsection{Causes internes de vulnérabilité}

Toute une école de pensée, essentiellement américaine, considère que la cause fondamentale de défaillance des entreprises réside dans le manque d'expérience ou l'incompétence des dirigeants (Argenti, 1976; Miller, 1977 ; Berryman, 1983 ; Peterson, Kozmetsky et Ridgway, 1983 ; Altman, 1983). L'explication d'Argenti sur le processus de défaillance en trois temps est typique de ce courant. Le premier stade se caractérise par la rigidité de la direction, en raison de la nature autocratique du chef d'entreprise, de la concentration du pouvoir sur sa seule personne, de compétences réduites à quelques domaines, de la faiblesse des services comptables et financiers, de l'absence de gestionnaires susceptibles de relayer la direction au sein de l'organisation... Tout cela restreint la capacité de la direction à recueillir l'information pertinente et à la traiter convenablement. Il en résulte, dans un deuxième stade, toute une série d'erreurs, qui ont pour but de pallier la dégradation de l'activité, mais qui ne font qu'aggraver la situation : détérioration des équilibres financiers, accroissement du volume de l'activité au détriment des marges... Ces erreurs amorcent le troisième stade au cours duquel apparaissent les symptômes de la faillite. 


\subsubsection{Causes externes de vulnérabilité}

Se référant à diverses études faites en France par le Crédit d'équipement des petites et moyennes entreprises (CEPME) qui font apparaître, comme cause première de la défaillance, l'incapacité des dirigeants dans moins de $10 \%$ des cas, Malecot $(1986,1988)$ se tourne vers l'analyse stratégique afin d'expliquer les faillites : déclin de la demande à l'entreprise, jeu des forces concurrentielles qui pèsent sur la rentabilité des actifs de la firme, nature et degré du risque supporté par l'entreprise (forte dépendance d'un marché, d'un client, d'un fournisseur, accès difficile et coûteux aux sources de financement) sont autant de raisons essentielles dans la défaillance.

On retrouve finalement, comme déclencheurs de la faillite, les mêmes éléments que ceux qui déclenchent l'émergence : le degré de compétence du dirigeant et une stratégie plus ou moins adaptée à l'environnement concurrentiel. La mise en évidence de ces éléments est à l'origine des méthodes de diagnostic de vulnérabilité.

\subsection{Le diagnostic de vulnérabilité}

Afin de prévoir et de prévenir la défaillance, divers auteurs ont proposé des méthodes de diagnostic de vulnérabilité utilisant des indicateurs synthétiques fondés sur des données quantitatives et aussi qualitatives.

La méthode de diagnostic de vulnérabilité la plus utilisée consiste à classer les populations d'entreprises en fonction du risque de défaillance apprécié par des données uniquement financières. Partant des travaux de Beaver (1966), Altman (1968) a utilisé l'analyse discriminante pour établir une fonction linéaire de ratios dont le score est largement diffusé et utilisé, malgré un pouvoir de prédiction contestable.

Quelques méthodes de diagnostic associent aux données quantitatives des informations qualitatives. Argenti (1977) complète l'analyse discriminante, par la méthode dite du "A score », construite autour de son modèle explicatif de défaillance, qui privilégie les facteurs de vulnérabilité comme les faiblesses de la direction, les insuffisances du système d'informations comptables et financières, l'incapacité à gérer le changement, la poursuite de la croissance au détriment de la rentabilité, ainsi que les erreurs de gestion tel un niveau trop élevé de risques d'exploitation et de risques financiers. Kœning (1985) cite la méthode de Chernet qui, dans une optique dynamique d'évolution de l'entreprise, propose huit indicateurs de synthèse permettant d'apprécier la cohérence, l'efficacité et la sécurité des choix effectués par l'entreprise dans les domaines financiers, humains et informationnels, avec un référentiel de 256 profils. 
Si l'application des méthodes qualitatives de diagnostic reste limitée faute de critères suffisamment précis, la démarche ne manque pas d'intérêt, en particulier pour le problème qui nous intéresse : l'établissement d'un diagnostic d'émergence. Par contre, la méthode des scores ne présente, elle, qu'un faible intérêt pour la détection de l'émergence, surtout lorsqu'il s'agit de création de PE ou TPE avec des données comptables souvent embryonnaires et sans historique.

En ce qui concerne l'émergence des entreprises, si la littérature en ce domaine est plus récente, il n'en existe pas moins des études intéressantes pouvant servir de base à un diagnostic d'émergence.

\section{Facteurs de développement et diagnostic d'émergence}

Les premières recherches sur le développement des PE et TPE ont porté sur les facteurs clés de succès (FCS) de la création d'entreprises ; elles ont permis d'établir des typologies de PE fort utiles pour l'établissement d'un diagnostic d'émergence.

\subsection{Les facteurs clés de succès de la création d'entreprise}

Afin d'isoler les FCS de la création d'entreprise, MacMillan, Siegel et Narashima (1985) ont étudié les critères utilisés par les sociétés de capital-risque pour évaluer un projet, critères relatifs à l'entrepreneur et aux compétences en management réunies autour du projet et critères relatifs à l'analyse de l'activité. Stuard et Abetti (1988) ramènent à trois les facteurs de la première catégorie : la personnalité, l'expérience ainsi que les capacités et la vision du créateur. Cooper, Dunkelberg et Woo (1988) ont recherché des corrélations entre la formation, l'expérience du créateur et le succès de la création, ainsi que des associations entre types d'entrepreneurs et types d'entreprises. Les facteurs de la deuxième catégorie sont les caractéristiques du marché, le degré d'innovation, la stratégie et l'organisation, éléments dont dépend le succès de la nouvelle entreprise selon Stuart et Abetti (1987) ; à cela s'ajoutent les facteurs de risque comme la nature du couple produit-marché, le type de processus de fabrication, l'importance de l'innovation (Masscarier et Rigaud, 1984 ; Rigaud, 1987 ; Ramus, 1989), ainsi que le manque de liquidités résultant d'une souscapitalisation ou d'une insuffisance de prévision (Fourcade, 1986 ; Neiswander et Drollinger, 1986). Plus récemment, Dubini (1989) s'est interrogé sur l'efficacité du processus de sélection basé sur ces facteurs. Livian et Marion (1991) ont testé, sur un échantillon de 11 entreprises, la valeur de pronostic de tels critères ; il apparaît que si l'existence d'une vision claire du projet concrétisée dans 
un plan d'affaires constitue un bon élément annonciateur du succès, le jugement sur les capacités personnelles de l'entrepreneur a parfois été démenti par les faits.

La prise en compte de ces facteurs permet une approche en termes de diagnostic ; ainsi Archer (1990) a proposé un diagnostic de prédémarrage, ce dernier étant défini par l'auteur comme : " la période qui précède le stade de création et d'installation ». Il s'agit d'établir et de préciser le degré de force ou de faiblesse des rapports de l'entreprise potentielle avec son environnement à partir de huit indicateurs de vulnérabilité $(1:$ barrières à l'entrée, 2 : fournisseurs, 3 : acheteurs, 4 : technologie, $5:$ gouvernement, $6:$ substituts (produits), 7 : substituts (intrants), 8 : distribution) évalués sur une échelle ordinale.

Une telle approche se révèle particulièrement intéressante pour notre recherche, laquelle est complémentaire de celle de Archer ; en effet nous nous intéressons à des entreprises déjà créées, qui se situent à la fin du stade de démarrage et qui se dirigent vers le stade de réussite. Le diagnostic d'émergence doit se faire de façon plus large, car il faut envisager non seulement la position de l'entreprise face à son environnement, mais aussi les capacités de son dirigeant et les potentialités de son organisation. Les études typologiques semblent alors particulièrement intéressantes pour faire ressortir les caractéristiques les plus adaptées à l'émergence.

\subsection{Les typologies de petites entreprises}

Nombre de chercheurs ont tenté d'établir des typologies opérationnelles de PME que Julien (1991) a recensées. Des typologies quantitatives basées sur le nombre d'employés, le chiffre d'affaires ou le montant des actifs, on est passé aux typologies qualitatives plus complexes; elles sont fondées sur le type de propriété (Deeks, 1973 ; Gelinier et Gaultier, 1974) ou elles introduisent l'évolution de la taille de l'entreprise (Liles, 1974 ; Filey et Aldag, 1978), la stratégie suivie par le direction (Cooper et Dulkenberg ; 1982, Carland et alii, 1984), les objectifs de l'entrepreneur (Marchesnay, 1988). Déjà au stéréotype de l'entrepreneur-artisan, Marchesnay (1985) avait opposé le nouvel entrepreneur qui est soit issu de la crise industrielle, soit un jeune créateur diplômé. En approfondissant les buts des dirigeants de petites entreprises, Marchesnay (1988) distingue l'entrepreneur PIC (pérennité, indépendance, croissance) analogue à l'entrepreneur-artisan, et l'entrepreneur CAP (croissance, autonomie, pérennité), opportuniste qui joue les turbulences de l'environnement pour lancer des affaires rentables ; le comportement du CAP est un des éléments déclencheurs de l'émergence. D'autres typologies sont fondées sur le cycle de vie de l'entreprise (Greiner, 1972 ; Churchill et Lewis, 1983 ; Vargas, 1984), schéma déterministe critiquable mais utile pour la compréhension du phénomène d'émergence, ou encore, pour les types et les opportunités de marché (Julien et Chicha, 1982)... 
Julien (1991) résume ces typologies en une typologie complexe globale, sur " continuum » allant de moins à plus, et composée de six éléments (1: dimension brute, $2:$ secteur, $3:$ marché, $4:$ organisation, $5:$ stratégie et objectifs, 6 : technologie et innovation). L'auteur pense que les PE et les TPE se trouveront plutôt dans les valeurs faibles, et les ME dans les valeurs fortes, mais avec des différences sur certains continua, en raison de la grande hétérogénéité des PME. Une telle approche peut être utilisée pour établir un diagnostic d'émergence en ordonnant les variables représentatives des éléments cités par Julien; les entreprises en émergence se situeront vraisemblablement vers la gauche dans les continua relatifs aux objectifs, au marché, à la technologie et à l'innovation.

En considérant toutes ces études et méthodes, nous sommes maintenant à même de proposer une méthode de diagnostic d'émergence.

\subsection{Le dlagnostic d'émergence}

Le diagnostic d'émergence doit faire apparaître les variables correspondant aux éléments moteurs de l'émergence qui, d'après notre étude précédente et les études typologiques sur les petites entreprises, sont :

- la perception stratégique du dirigeant et son comportement face aux opportunités et aux risques de l'environnement ;

- le caractère de la stratégie ;

- les composants de la stratégie : complexité commerciale, complexité technologique, sous-traitance, politique d'investissement, politique de coopération...

Ces variables sont essentiellement qualitatives; nombre d'entre elles sont appréciées au travers des réponses du dirigeant, ce qui comporte un risque marqué de subjectivité. Ce risque, inévitable dans ce type d'enquêtes, est cependant atténué par les observations de l'enquêteur. L'analyse étant faite sur un continuum, les variables sont ordonnées de façon croissante grâce à l'utilisation d'échelles à cinq niveaux.

En raison de l'hétérogénéité des petites entreprises, il est indispensable de mesurer le plus grand nombre possible de variables dans les domaines retenus, de faire apparaître les variables les plus caractéristiques de l'échantillon d'entreprises, puis de déterminer des classes homogènes de firmes dont l'étude permettra de préciser les facteurs d'émergence. Pour cela, l'analyse factorielle constitue un outil indispensable : l'analyse des correspondances multiples (ACM) met en évidence les modalités des variables les plus significatives ainsi que les firmes concernées, tandis que la classification ascendante hiérarchique $(\mathrm{CAH})$ regroupe ces entreprises en classes homogènes. 
Telle est la base du diagnostic d'émergence proposé que nous allons appliquer à un échantillon de PE et TPE dont la nature de l'activité et le caractère des dirigeants laissent présager des possibilités de développement futur.

\section{La mise en œuvre du diagnostic d'émergence}

Dans un canton (circonscription territoriale en France qui englobe la commune et ses environs) de moyenne importance du Maine-et-Loire, confronté depuis plusieurs années à des difficultés économiques, il s'agissait de revitaliser le tissu des petites entreprises, en sélectionnant des firmes à possibilité d'émergence auxquelles une aide publique au développement pouvait être accordée. Deux cents entreprises inscrites au répertoire des Métiers étaient concernées. Parmi celles-ci, il apparaissait très vite qu'un bon nombre ne présentaient aucune possibilité de croissance (entreprises artisanales à activité traditionnelle, à clientèle uniquement locale ou avec un dirigeant âgé ne désirant ni développer son activité, ni rechercher de nouveaux clients), alors que certaines présentaient des potentialités réelles d'émergence ou étaient en plein processus d'émergence.

La sélection a été réalisée par deux étudiants et par l'auteur, en collaboration avec des professionnels de la Chambre des Métiers du Maine-et-Loire, au moyen d'entretiens téléphoniques avec chacun des 200 dirigeants. Les renseignements demandés (regroupés dans un guide d'entretien) étaient les suivants :

1. raison sociale de l'entreprise,

2. forme juridique actuelle,

3. date de création de l'entreprise,

4. activité principale,

5. activités secondaires,

6. existence d'un produit original, d'un savoir-faire ou d'une spécialité susceptibles d'être développés,

7. conditions nécessaires au développement,

8. zone habituelle de clientèle,

9. possibilités d'extension du marché actuel.

Vingt-huit entreprises ont été ainsi retenues en tenant compte sur la base de l'intention du chef d'entreprise de développer son affaire, de l'existence d'un produit ou d'un savoir-faire original, d'une activité sur des marchés nouveaux et porteurs (photo-composition, climatisation...) ou peu concurrencés (armurerie, récupération de matériaux anciens...). Le risque de subjectivité dans la sélection a été limité par le recours à deux équipes : les professionnels qui 
connaissent les entreprises, mais qui peuvent avoir des a-priori ; les universitaires qui travaillent avec une approche plus scientifique, mais qui connaissent moins le marché.

Toutefois, avant d'effectuer un diagnostic d'émergence fondé sur l'analyse multidimensionnelle des variables, nous présenterons les entreprises choisies, les variables et leur traitement statistiques, puis nous ferons un bref résumé de l'analyse unidimensionnelle de ces variables.

\subsection{Les caractéristiques générales de l'échantillon}

Les caractéristiques générales des 28 entreprises sélectionnées sont résumées dans le tableau suivant :

TABleAu 1

\section{Caractéristiques générales des entreprises}

(Nombre d'entreprises)

\begin{tabular}{|c|c|c|c|c|c|}
\hline SECTEUR & Alimentation & Bâtiment & Production & Réparation & Services \\
\cline { 2 - 4 } & $\frac{9}{4}$ & $\frac{9}{4}$ & $\frac{2}{2}$ \\
\hline
\end{tabular}

\begin{tabular}{|c|c|c|c|c|c|}
\hline \multirow{2}{*}{$\mathrm{A} G E$} & $<4$ ans & $4-8$ ans & $9-11$ ans & $12-20$ ans & $\frac{21-31 \text { ans }}{6}$ \\
\cline { 2 - 4 } & 5 & $\frac{5}{6}$ & $\frac{6}{3}$ \\
\hline
\end{tabular}

\begin{tabular}{|c|c|c|c|c|c|}
\hline ÂGE DU & $20-29$ ans & $30-39$ ans & $40-49$ ans & 50 - 59 ans & 60 ans et plus \\
\hline DIRIGEANT & 3 & 11 & 9 & 5 & 0 \\
\hline
\end{tabular}

\begin{tabular}{|c|c|c|c|c|c|}
\hline ORIGINE & creation & reprise & & & \\
\hline & 14 & $\frac{14}{4}$ & & & \\
\hline
\end{tabular}

\begin{tabular}{|c|c|c|c|c|c|}
\hline $\begin{array}{c}\text { FORME } \\
\text { JURIDIQUE }\end{array}$ & $\begin{array}{c}\text { Entreprise } \\
\text { individuelle }\end{array}$ & SARL & SA & & \\
\hline 11 & $\frac{15}{2}$ & $\frac{2}{25}$ & \\
\hline
\end{tabular}

\begin{tabular}{|c|c|c|c|c|c|}
\hline EFFECTIF & $<6$ & $\frac{6 \text { à } 10}{10}$ & $\frac{11 \text { à } 15}{7}$ & $\frac{16 \text { à } 20}{4}$ & $\frac{21 \text { à } 25}{3}$ \\
\hline
\end{tabular}


Cet échantillon appelle deux remarques :

L'âge moyen des entreprises est de 11,5 ans, avec un minimum de 1 an et un maximum de 31 ans, ce qui nous donne un continuum d'âge étendu. Nous avons défini l'émergence comme un processus dynamique consistant dans le passage de la phase de démarrage (qui peut durer de 2 à 5 ans) à la phase de réussite-croisière ou de réussite-développement. Certaines entreprises apparaissent donc au début de la phase de démarrage, d'autres en pleine phase de réussite, de sorte que nos observations portent sur des étapes variées du processus d'émergence. En fait, le processus n'est pas aussi régulier que pourraient le faire penser ces considérations ; Churchill et Lewis (1983) ont bien analysé ce phénomène. À l'issue du démarrage qui s'effectue selon eux en deux stades, l'existence et le maintien, le troisième stade, la réussite, peut prendre deux formes : la réussite-désengagement ou la réussite-expansion. Lors de la réussitedésengagement, l'entreprise a atteint une taille et une pénétration du marché suffisantes pour obtenir des bénéfices égaux ou supérieurs à la moyenne; elle peut se maintenir indéfiniment à ce stade, tant que les modifications de l'environnement ne nuisent pas à son créneau de marché et qu'une gestion maladroite ne réduit pas son avantage compétitif. Il est vraisemblable que cela soit le cas de plusieurs entreprises de plus de 5 ans dans notre échantillon, en raison d'un environnement concurrentiel assez stable dans une région en proie à un certain marasme économique ; elles ont un ou plusieurs DAS dans lesquels elles font preuve d'une compétitivité suffisante. Si l'environnement se modifie en profondeur, soit elles reviennent au stade de maintien, soit elles réagissent et passent au stade de réussite-expansion. L'autre forme possible est la réussite-expansion, grâce au recours aux fonds propres et à la capacité d'emprunt pour financer la croissance, et avec la mise en œuvre d'une véritable planification stratégique. La réussite-expansion débouche normalement sur l'essor et la possibilité de devenir une grande entreprise ; mais, là encore, des replis sont possibles, de l'expansion ou l'essor vers le désengagement, de même que le maintien d'un point d'équilibre lorsqu'un certain niveau de réussite est atteint.

La moitié des entreprises de notre échantillon est issue de création et l'autre moitié de reprise ; en effet, l'arrivée d'un nouveau dirigeant-propriétaire peut être un facteur de passage du démarrage à la réussite ou encore de la réussite-désengagement à la réussite-expansion et à l'essor.

C'est l'étude des variables qui va permettre de connaître le comportement stratégique des entreprises de l'échantillon.

\subsection{Les varlables et le traitement statistique}

Les variables retenues pour le diagnostic d'émergence ont été mesurées à la suite d'un entretien directif, mené à l'aide d'un questionnaire détaillé (17 pages), et 
complété par un entretien non directif avec chacun des 28 chefs d'entreprise. Ces variables font apparaître de nombreux caractères :

- Les caractéristiques importantes de l'entreprises pour juger son niveau actuel :

1. Caractères généraux (âge, origine, forme juridique);

2. Effectif (actuel et variation sur les deux dernières années);

3. Performances actuelles (taux de croissance du chiffre d'affaires, type de croissance, rentabilité) ; les performances ont été volontairement limitées aux éléments les plus aisément mesurables (croissance, rentabilité) que nous pouvions obtenir pour toutes les entreprises ; a été ajouté le mode de croissance, interne ou externe, car les PE adoptent volontiers un mode de croissance « en grappes » pouvant aboutir à la constitution d'hypogroupes.

- Le caractère général de la stratégie appréhendée au travers de :

4. Perspectives de développement (taux de croissance envisagé, expansion du marché actuel, possibilités de produits nouveaux, perspectives d'investissement).

- Les composants de la stratégie :

5. Organisation de la production (complexité technologique, part de l'activité de donneur d'ordre, part de la sous-traitance) ;

6. Produits et marchés de l'entreprise (diversité de l'activité, situation du marché, répartition des clients, nombre de produits leaders) ;

7. Entreprise et environnement : degré d'informatisation, politique de coopération, situation par rapport aux banques. L'informatisation est la condition d'un système pertinent d'informations sur l'environnement ; la coopération est un facteur important de la stratégie des PE qui se développent à l'intérieur et à l'aide de réseaux complexes.

- Le caractère et les capacités stratégiques du dirigeant :

8. Attitude du dirigeant de l'entreprise (participation à la décision, attitude face à l'environnement et au risque) ;

9. Perception stratégique du dirigeant (importance accordée à divers objectifs stratégiques).

Nombre de ces variables ont été déterminées de la même manière que dans notre étude de 1988 : par agrégation de scores; il en est ainsi, en particulier de la diversité de l'activité (DIV) (nombre de DAS et étendue de la gamme de 
produits), de la complexité technologique (TEC) (diversité technologique et types de technologie) Capiez, 1988, p. 140) et des caractéristiques psychologiques des dirigeants : participation à la décision (PPD) (agrégation de 5 affirmations) (cf. étude citée, p. 134), attitude réactive ou proactive face à l'environnement (AEN) (agrégation de 2 affirmations), attitude face au risque (RIS) (agrégation de 3 affirmations) (cf. étude citée, p. 136).

Les modalités de ces variables ont été, pour la plupart, ordonnées de 1 à 5 , soit 191 modalités (voir tableaux 2 et 3 , pages suivantes).

L'analyse unidimensionnelle de ces variables donne un aperçu général de la stratégie mise en œuvre par les entreprises de l'échantillon.

\subsection{L'analyse unidimensionnelle des varlables}

Comme dans beaucoup de PE et TPE, le dirigeant-propriétaire (DP) (seul propriétaire dans $39 \%$ des cas, majoritaire dans $25 \%$ et majoritaire avec la famille dans $36 \%$ des cas) centralise la plupart des décisions. En matière de participation à la décision (PPD), $68 \%$ des DP ont un score de 1 et $2 ; 25 \%$, de 3 et seulement $7 \%$, de $4 ; 71 \%$ des DP déclarent n'avoir jamais ou rarement de problèmes avec leurs salariés. Vis-à-vis de l'environnement, les DP considèrent leur attitude (AEN) comme essentiellement proactive ( $21 \%$ ont un score de 3 , $54 \%$ de 4 et $25 \%$ de 5 ) et, en matière de risques (RIS), la tendance se situe vers la « recherche des risques », mais raisonnée (39\% ont un score de 1 et $2,54 \%$ de 3 et $7 \%$ de 5). Les objectifs considérés comme prioritaires par les DP (perception stratégique) sont en ordre décroissant, la productivité (PTE) et la flexibilité (FLX) (score moyen de 4), les parts de marché (MAR), les liquidités (LIQ) et la croissance (CRO) (score moyen de 3,8), l'indépendance (IND) (score moyen de 3). Si l'on ajoute que $50 \%$ des DP ont atteint au moins le niveau du brevet professionnel ou du baccalauréat (dont $18 \%$ avec une formation supérieure telles BTS ou IUT et $11 \%$ avec une formation spécialisée) et que $36 \%$ possèdent un CAP ou un BEP, on est très proche du type d'entrepreneur CAP défini par Marchesnay.

Le caractère général de la stratégie apparaît orienté vers la croissance (stratégie délibérée pour $50 \%$ des entreprises, stratégie favorisant plutôt la croissance pour $21 \%$ ), croissance essentiellement interne (71\% des entreprises, $18 \%$ ayant acquis ou créé une autre entreprise et $11 \%$ deux autres), avec un recours significatif à l'endettement ( $57 \%$ des entreprises considèrent leur endettement comme élevé et $11 \%$ utilisent uniquement l'autofinancement). Cependant, bon nombre ont du mal à chiffrer le taux de croissance souhaité (TX) et donnent une estimation moyenne faible (14\%) par rapport à leur croissance réelle (CRC) sur 
les trois dernières années (24\% par an en moyenne). Plus de la moitié des entreprises (54\%) envisage un développement futur de l'activité (SIT), avec des produits nouveaux (NEW) (79 \% des entreprises). En matière d'investissement, $86 \%$ des entreprises ont réalisé des investissements de production depuis deux ans (INV) et $58 \%$ envisagent des investissements dans les deux ans à venir (IVF), afin d'accroître la capacité de production des produits actuels, élargir la gamme de produits et s'adapter à l'évolution technologique.

En ce qui concerne les composants de la stratégie, la complexité technologique (TEC) est faible pour $43 \%$ des entreprises (surtout dans le bâtiment et l'alimentaire), moyenne pour $36 \%$ et forte pour $21 \%$ (en particulier les soustraitants de grands groupes industriels). L'activité (DIV) apparaît moyennement diversifiée (2 à 3 DAS pour $79 \%$ des entreprises), avec une gamme moyenne de produits, un ou deux produits leaders (LEA) en moyenne, un marché (SMA) considéré comme en croissance par $61 \%$ des entreprises, et une clientèle (REP) s'étendant à la région pour $71 \%$ des entreprises, à la France entière pour $18 \%$ et à l'Europe pour $4 \%$. L'informatisation (INF) est nulle pour $36 \%$ des entreprises, limitée pour $53 \%$ et complète pour $11 \%$ des entreprises qui l'utilisent systématiquement pour la prise de décision. Enfin, la politique de coopération avec d'autres entreprises locales (ENT) paraît au premier abord limitée, puisque $25 \%$ seulement des entreprises ont adopté une stratégie d'alliance (pour la réalisation de chantiers importants dans le bâtiment) et $7 \%$ font partie d'un GIE. Mais la coopération réelle est plus importante ; la moitié des entreprises de l'échantillon sont sous-traitantes à des degrés divers (dont 3 à plus de $70 \%$ ) et coopèrent avec des groupes industriels; en outre $21 \%$ des entreprises « externalisent » une partie de leur activité (de 10 à $30 \%$ pour deux d'entre elles), ce qui leur permet d'augmenter le chiffre d'affaires sans modifier leur taille. Enfin, $57 \%$ des entreprises sont très endettées (SBQ) et sont ainsi en relation étroite avec les réseaux financiers (groupes bancaires, sociétés de conseil, institutions régionales), comme l'a montré Belletante (1991). Ces entreprises, insérées dans des réseaux complexes, sont ainsi rendues fortement interactives par le maillage d'activités variées qui se révèlent complémentaires.

Mais ce sont les relations entre ces différentes variables qui sont particulièrement intéressantes et qui permettent d'établir le diagnostic d'émergence.

\subsection{L'analyse multidimensionnelle des variables : le dlagnostic d'émergence}

Après avoir commenté les relations entre quelques variables significatives prises deux à deux, nous présenterons les résultats de l'analyse factorielle qui constituent le diagnostic d'émergence. 
TABleAU 2

Modalités des variables

\begin{tabular}{|c|c|c|c|c|}
\hline $\begin{array}{l}\text { Sootour d'aollulto : SEC } \\
\text { ALIM : Alimentation } \\
\text { BAT : Bdimont } \\
\text { PROD : Production } \\
\text { REPA : RGparation } \\
\text { SERV : Services }\end{array}$ & 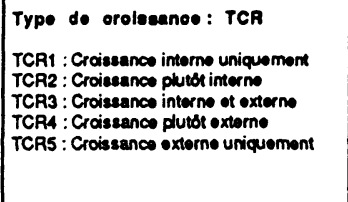 & 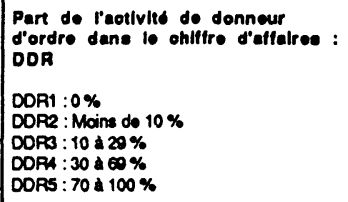 & 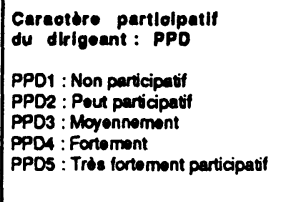 & $\begin{array}{l}\text { Importanoe do la lloxlbilite: FLX } \\
F L X 1 d F L X 5\end{array}$ \\
\hline $\begin{array}{l}\text { Age do l'ontroprise : AGE } \\
\text { AGE1 : Mains do } 4 \text { ans } \\
\text { AGE2 : } 4 \text { a } 8 \text { ans } \\
\text { AGES : }: 12 \text { ans } \\
\text { AGE4 : } 12 \text { \& } 20 \text { ans } \\
\text { AGES : } 21 \text { \& } 31 \text { ans }\end{array}$ & 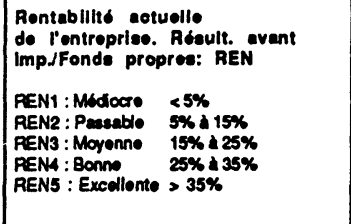 & 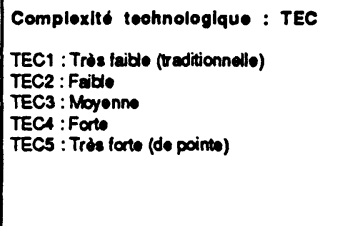 & 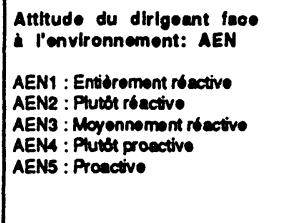 & 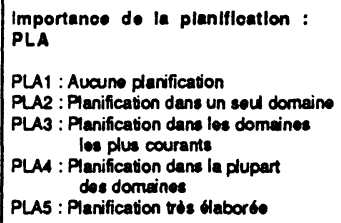 \\
\hline $\begin{array}{l}\text { Ag0 du dirlgoent : AGD } \\
\text { AGD1 : } 20-29 \text { ans } \\
\text { AGD2 : } 30-39 \text { ans } \\
\text { AGDS : } 40-49 \text { ans } \\
\text { AGD4 :50-50 ans } \\
\text { AGDS: } 60 \text { et plus }\end{array}$ & $\begin{array}{l}\text { SItuation future onvieagde: SIT } \\
\text { STT1 : Maintion } \\
\text { SIT2 : D6velopperment }\end{array}$ & $\begin{array}{l}\text { Inveatlesemonte de produotion } \\
\text { róalleco dopule doux ane: INV } \\
\text { INV1 : Non } \\
\text { INV2:Oui }\end{array}$ & 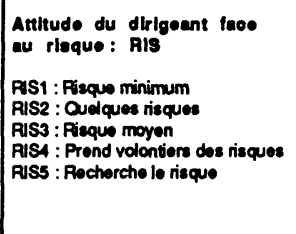 & 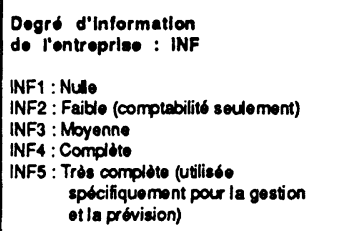 \\
\hline $\begin{array}{l}\text { OrIgine de l'ontroprles : ORI } \\
\text { ORI1 : Croation } \\
\text { ORI2 : Roprise }\end{array}$ & 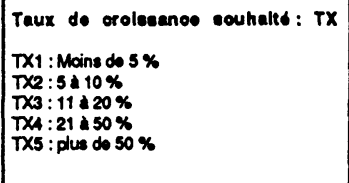 & 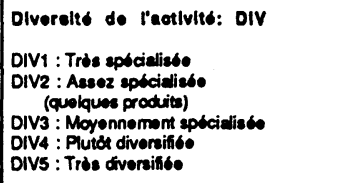 & 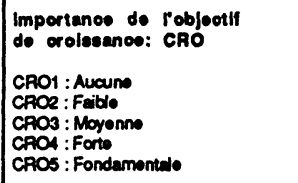 & 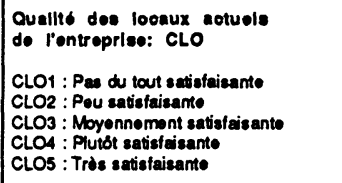 \\
\hline
\end{tabular}




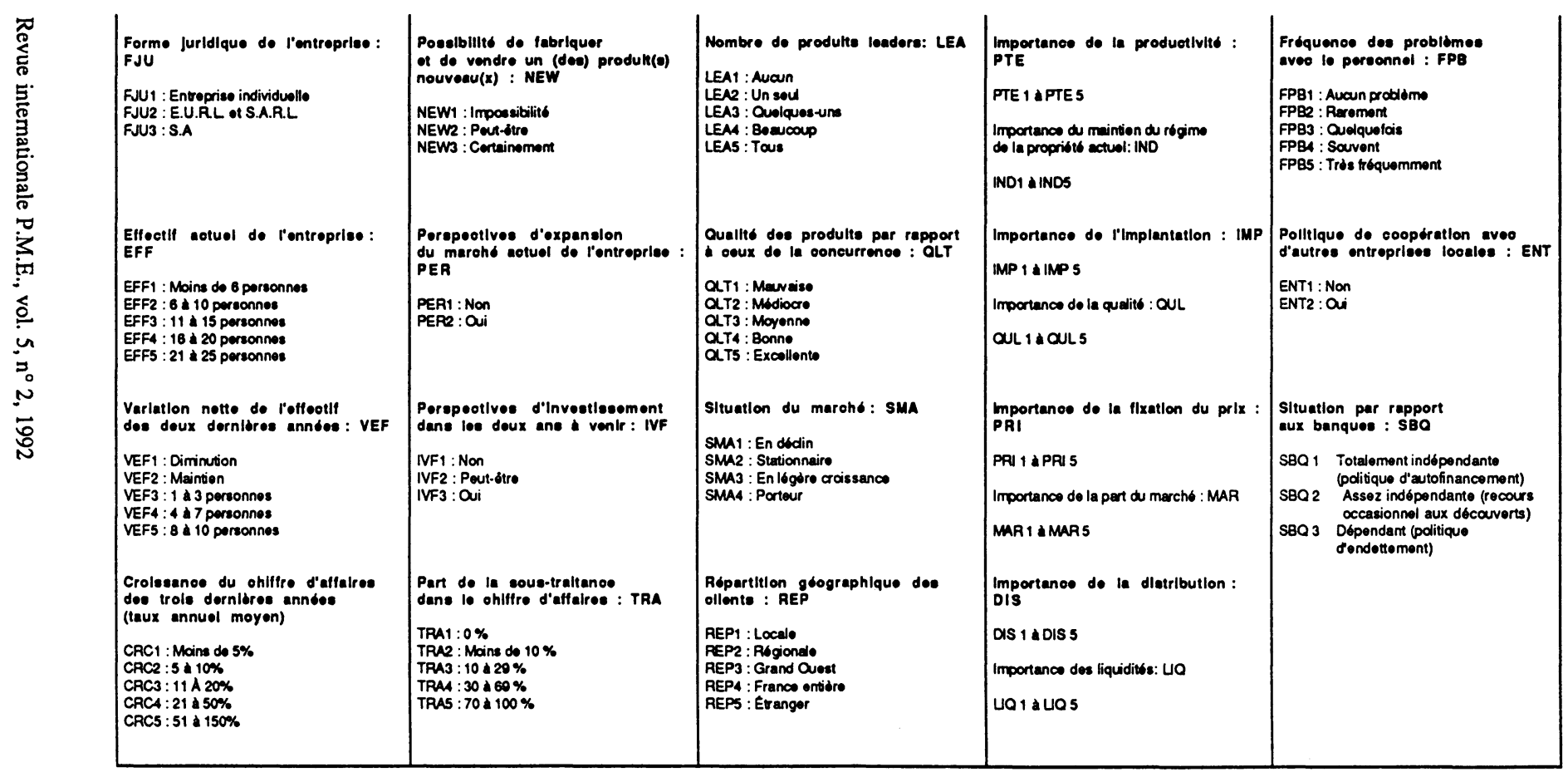


TABLEAU 3

Variables et code des modalités

\section{Varlables}

1 Caracteres généraux de l'entreprlse Sectour

Age de l'entreprise

Àge du dirigeant

Origine de l'entreprise

Forme juridique de l'entreprise

\section{Effectlf de l'entreprise}

- Effectif actuel de l'entreprise

- Variation de l'effectif ces deux dernières années

3 Performances actuelles de l'entroprise

- Croissance du chiffre d'affaires des trois dernières années

- Type de croissance

- Rentabilité actuelle de l'entreprise

\section{Perspectlves de développement}

Situation future envisagée

- Taux de croissance souhaité

- Possibilité de produits nouveaux

- Perspectives d'expansion du marché actuel de l'entreprise

Perspectives d'investissements prochains

5 Organlsation de la production

Part de la sous-traitance

- Part de l'activité de donneur d'ordres

Complexité tochnologique

- Investissements de productin récents
Codes ordonnés de façon crolssante partir de 1

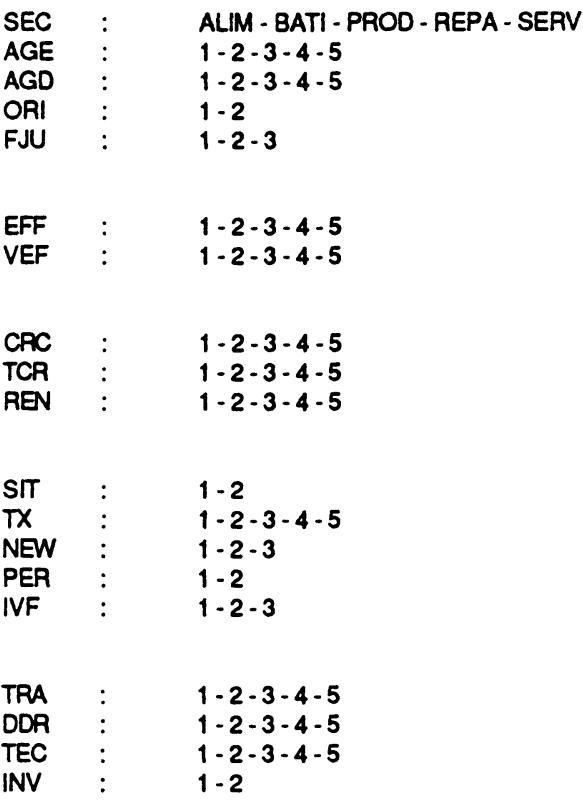




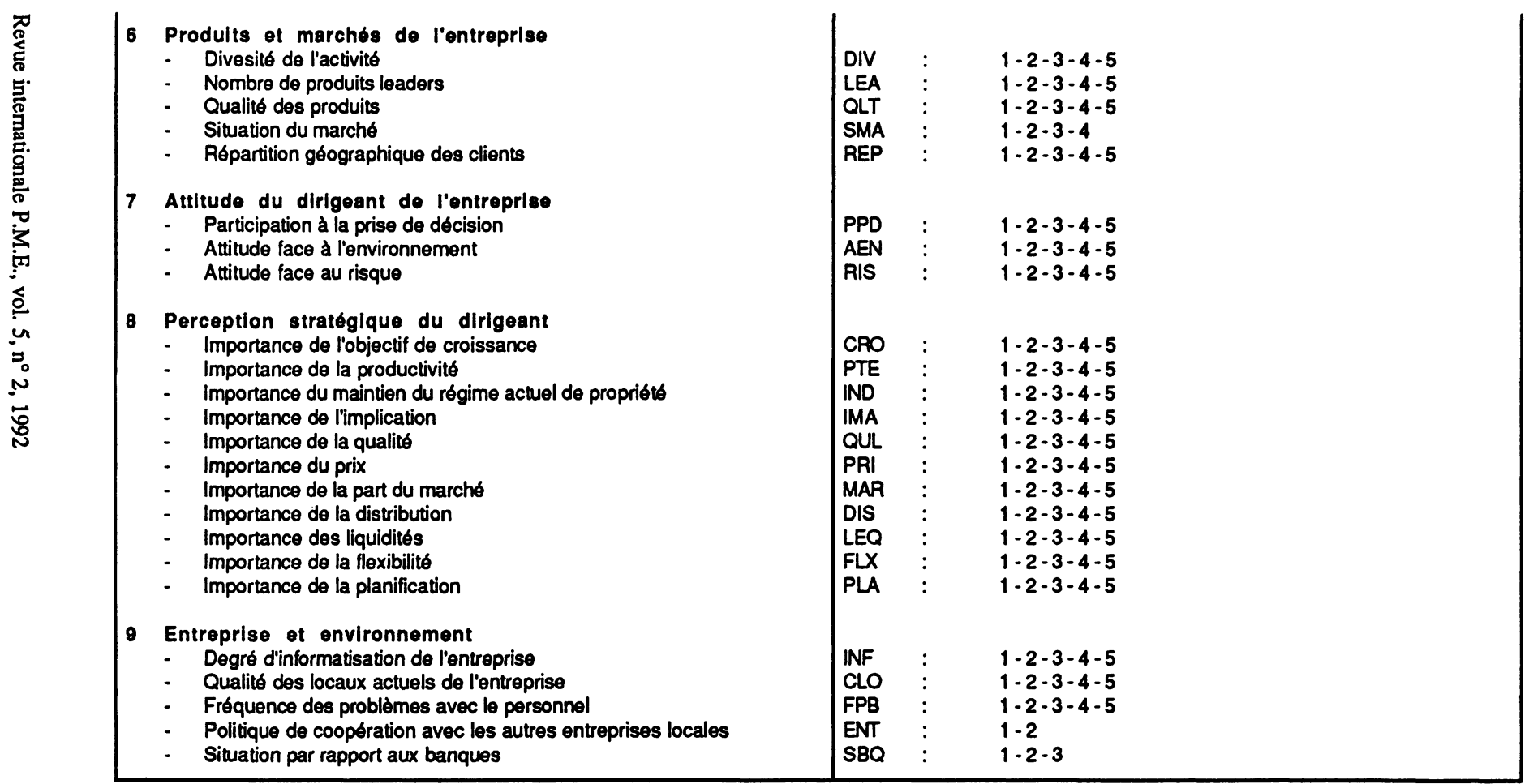




\subsubsection{Les relations entre quelques variables significatives}

Parmi les nombreuses variables calculées, certaines semblent particulièrement significatives et le calcul du chi-deux entre celles-ci prises deux à deux fait apparaître des liaisons intéressantes (voir tableau 4, page suivante).

- Ce sont les dirigeants les plus proactifs face à l'environnement (AEN) qui recherchent la plus forte croissance, la croissance attendue (TX) étant elle-même liée à la croissance actuelle (CRC).

- Ce sont les entreprises qui accordent le plus d'importance à leur part de marché (MAR) qui ont la possibilité de proposer des produits nouveaux (NEW) et les entreprises qui ont le plus de produits leaders (LEA) qui créent le plus d'emplois (VEF).

- Les entreprises qui envisagent des investissements dans les deux années à venir (IVF) sont celles qui s'intéressent le plus à leur part de marché (MAR), ont le plus de produits leaders (LEA) et envisagent le plus de produits nouveaux (NEW).

Le recours à l'analyse factorielle est indispensable pour synthétiser toutes ces relations ; cette analyse sera menée en deux temps : d'abord l'ACM fera ressortir axe par axe les variables et leurs modalités les plus importantes afin de regrouper ou d'opposer les entreprises ; puis, la $\mathrm{CAH}$ rassemblera les entreprises en classes homogènes résumant les différents axes.

\subsubsection{L'analyse factorielle des correspondances multiples}

En raison du nombre important des modalités traitées et de la grande dispersion des données qui en résulte, il apparait nécessaire d'étudier les six premiers axes qui représentent respectivement $7,66 \%, 6,74 \%, 6,43 \%, 6,16 \%, 5,57 \%$ et $5,15 \%$ de l'inertie du nuage, soit un cumul de $37,71 \%$. On s'aperçoit immédiatement que l'axe 1 est formé essentiellement par deux entreprises très semblables, l'entreprise 06 et l'entreprise 07, caractérisées par ces points aberrants (FLX2, AGDI, TCR3, MAR5, REN1, AGE1) : il s'agit de deux entreprises jeunes, avec de jeunes dirigeants, au taux de croissance important (CRC4 et CRC5), avec un mode de croissance à la fois interne et externe, mais à faible rentabilité, moyennement informatisées (INF3), dont les dirigeants accordent une importance fondamentale à leur part de marché mais moins à la flexibilité.

Ces deux individus, en raison de leur originalité, se distinguent fortement de la structure moyenne et risquent de la masquer par leur importance. Aussi est-il bon de les mettre en éléments supplémentaires et d'effectuer l'analyse sur les 26 autres entreprises. 


\section{TableaU 4}

Relations entre les variables les plus significatives : chi-deux

\begin{tabular}{|c|c|c|c|c|c|c|c|c|c|c|c|c|c|}
\hline & $T X$ & CAC & VEF & TCR & REN & $F L X$ & LIQ & MAR & NEW & LEA & IVF & AEN & RIS \\
\hline$T X$ & & & & & & & & & & & & & \\
\hline CRC & $\begin{array}{r}\mathbf{x x x} \\
33,75 \\
\cdot(16) \\
\end{array}$ & & & & & & & & & & & & \\
\hline VEF & $\begin{array}{r}13,43 \\
\cdot \quad 16) \\
\end{array}$ & $\begin{array}{r}21,97 \\
\cdot(16) \\
\end{array}$ & & & & & & & & & & & \\
\hline TCR & $\begin{array}{c}x X \\
25,88 \\
+(12) \\
\end{array}$ & $\begin{array}{r}12,44 \\
-(12) \\
\end{array}$ & $\begin{array}{r}10 \\
\times(12) \\
\end{array}$ & & & & & & & & & & \\
\hline REN & $\begin{array}{r}16,15 \\
+\quad 12) \\
\end{array}$ & $\begin{array}{r}12,9 \\
\cdot \quad(12) \\
\end{array}$ & $\begin{array}{r}11,64 \\
+\quad(12) \\
\end{array}$ & $\begin{array}{c}10,34 \\
\cdot(9) \\
\end{array}$ & & & & & & & & & \\
\hline$F L X$ & $\begin{array}{r}15,04 \\
+\quad(16) \\
\end{array}$ & $\begin{array}{r}18,77 \\
\cdot(16) \\
\end{array}$ & $\begin{array}{l}20,48 \\
\cdot(16) \\
\end{array}$ & $\begin{array}{r}15,87 \\
\cdot(12) \\
\end{array}$ & $\begin{array}{l}17,11 \\
\cdot(12) \\
\end{array}$ & & & & & & & & \\
\hline LIO & $\begin{array}{r}15,82 \\
\cdot \quad 12) \\
\end{array}$ & $\begin{array}{r}9,93 \\
+(12) \\
\end{array}$ & $\begin{array}{c}x \\
20,3 \\
\cdot(12) \\
\end{array}$ & $\begin{array}{r}9,51 \\
. \quad(9) \\
\end{array}$ & $\begin{array}{r}8,9 \\
-(9) \\
\end{array}$ & $\begin{array}{r}7,82 \\
-(12) \\
\end{array}$ & & & & & & & \\
\hline MAR & $\begin{array}{r}11,65 \\
+\quad 12) \\
\end{array}$ & $\begin{array}{r}12,05 \\
+(12) \\
\end{array}$ & $\begin{array}{r}11,77 \\
\cdot(12) \\
\end{array}$ & $\begin{array}{c}x x \\
20,05 \\
\cdot(9) \\
\end{array}$ & $\begin{array}{c}11,32 \\
\cdot(9) \\
\end{array}$ & $\begin{array}{r}13,92 \\
+(12) \\
\end{array}$ & & & & & & & \\
\hline NEW & $\begin{array}{r}4,63 \\
-\quad(8) \\
\end{array}$ & $\begin{array}{r}5,67 \\
\cdot \quad(8) \\
\end{array}$ & $\begin{array}{r}7,28 \\
\cdot(8) \\
\end{array}$ & $\begin{array}{r}4,48 \\
-\quad(6) \\
\end{array}$ & $\begin{array}{r}2,61 \\
\cdot(6) \\
\end{array}$ & $\begin{array}{r}9,84 \\
\cdot(8) \\
\end{array}$ & $\begin{array}{r}3,11 \\
\cdot(6) \\
\end{array}$ & $\begin{array}{c}x x \\
14,99 \\
\cdot(6) \\
\end{array}$ & & & & & \\
\hline LEA & $\begin{array}{r}8,82 \\
-\quad(12) \\
\end{array}$ & $\begin{array}{r}14,84 \\
\times(12) \\
\end{array}$ & $\begin{array}{c}x x x x \\
22,26 \\
\cdot(12) \\
\end{array}$ & $\begin{array}{c}13,29 \\
\cdot(9) \\
\end{array}$ & $\begin{array}{r}9,06 \\
\cdot(9) \\
\end{array}$ & $\begin{array}{r}9,5 \\
-(12) \\
\end{array}$ & $\begin{array}{c}12,97 \\
\cdot(9)\end{array}$ & $\begin{array}{l}13,86 \\
\cdot(9) \\
\end{array}$ & $\begin{array}{r}8,67 \\
\cdot \quad 6) \\
\end{array}$ & & & & \\
\hline INF & $\begin{array}{r}6,7 \\
-\quad(8) \\
\end{array}$ & $\begin{array}{r}6,94 \\
\cdot(8) \\
\end{array}$ & $\begin{array}{r}7,3 \\
. \quad 8 \\
\end{array}$ & $\begin{array}{c}5 \\
. \\
.\end{array}$ & $\begin{array}{r}5,86 \\
\cdot(6) \\
\end{array}$ & $\begin{array}{r}10,79 \\
\cdot(8) \\
\end{array}$ & $\begin{array}{l}4383 \\
\cdot(6) \\
\end{array}$ & $\begin{array}{r}x x \\
13,98 \\
\cdot(6) \\
\end{array}$ & $\begin{array}{r}\mathbf{x x x x} \\
15,66 \\
\cdot(4) \\
\end{array}$ & $\begin{array}{l}x \times x x \\
22.88 \\
\cdot(6) \\
\end{array}$ & & & \\
\hline AEN & $\begin{array}{c}x \times x \\
17,27 \\
+(6) \\
\end{array}$ & $\begin{array}{r}19,37 \\
+\quad(16) \\
\end{array}$ & $\begin{array}{r}20,95 \\
-(16) \\
\end{array}$ & $\begin{array}{c}\mathbf{x x} \\
21,67 \\
\cdot(12) \\
\end{array}$ & $\begin{array}{r}16,59 \\
\cdot(12) \\
\end{array}$ & $\begin{array}{r}23,24 \\
*(16) \\
\end{array}$ & $\begin{array}{r}13,56 \\
\cdot(12) \\
\end{array}$ & $\begin{array}{r}10,84 \\
\cdot(12) \\
\end{array}$ & $\begin{array}{r}8,8 \\
. \quad(8) \\
\end{array}$ & $\begin{array}{r}18.44 \\
\cdot(12) \\
\end{array}$ & $\begin{array}{r}3,03 \\
-\quad(4) \\
\end{array}$ & & \\
\hline RIS & $\begin{array}{r}19,16 \\
\cdot(16) \\
\end{array}$ & $\begin{array}{r}8,78 \\
-\quad 16) \\
\end{array}$ & $\begin{array}{r}11,84 \\
\cdot(16) \\
\end{array}$ & $\begin{array}{r}13,72 \\
\cdot(12) \\
\end{array}$ & $\begin{array}{r}4,43 \\
-\quad 12) \\
\end{array}$ & $\begin{array}{c}\mathbf{x x} \\
31,13 \\
\cdot(16) \\
\end{array}$ & $\begin{array}{r}7,79 \\
\cdot(12) \\
\end{array}$ & $\begin{array}{r}12,41 \\
-(12) \\
\end{array}$ & $\begin{array}{r}3,92 \\
\cdot(8) \\
\end{array}$ & $\begin{array}{r}15,55 \\
\cdot(12) \\
\end{array}$ & $\begin{array}{r}8,4 \\
+\quad 8) \\
\end{array}$ & $\begin{array}{l}19,04 \\
\cdot(16)\end{array}$ & \\
\hline
\end{tabular}

* Le nombre de degrés de liberté figure entre parenthèses

$$
\begin{aligned}
X & \text { significatif pour } X=0,1 \\
X X & \text { significatif pour } X=0,05 \\
X X X & \text { significatif pour } X=0,01 \\
X X X X & \text { significatif pour } X=0,005
\end{aligned}
$$


Avec une ACM sur 26 entreprises et les entreprises 06 et 07 en éléments supplémentaires, les six premiers axes représentent maintenant, respectivement, $7,19 \%, 7,13 \%, 6,64 \%, 6,02 \%, 5,67 \%$ et $5,54 \%$ de l'inertie du nuage, soit un cumul de $38,18 \%$ très proche du précédent. La représentation graphique des variables les plus contributives des deux premiers axes apparaît plus régulière, avec un effet Guttman plus prononcé. Ce sont les différents axes de l'ACM sur 26 entreprises que nous allons commenter maintenant.

\section{Le premier axe factoriel}

Les variables les plus contributives de l'axe 1 (LEA, EFF, FLX, IVF, ENT, MAR, AEN) voient leurs modalités les plus significatives situées soit sur la partie positive, soit sur la partie négative de l'axe. Sont ainsi séparées les entreprises à effectif important (EFF4 et EFF5), avec des perspectives d'investissement proches (IVF3) et quelques produits leaders (LEA3), des dirigeants proactifs face à l'environnement (AEN5), à bonne perception stratégique en matière de part de marché (MAR4, MAR5), de flexibilité (FLX5) et qui coopèrent volontiers avec d'autres entreprises locales (ENT2), comme les entreprises 13 et 14 d'une part, et, d'autre part, les entreprises à effectif plus faible (EFF2), incertaines quant à leurs investissements (IVF2) et sans produits leaders (LEA1), avec des dirigeants moins proactifs (AEN3), moins concernés par la stratégie (MAR3, FLX2 et FLX3) et non coopératifs (ENT1), comme les entreprises 04 et 20 .

\section{Le deuxième axe factoriel}

Les variables contributives, plus nombreuses que sur l'axe 1 (VEF, CRC, INF, PPD, FJU, MAR, LEA, REP, EFF), ont des modalités réparties, pour les valeurs élevées, sur la partie positive de l'axe et, pour les valeurs faibles, sur la partie négative. Sont ainsi distinguées, d'une part, les entreprises à forme sociétaire (FJU2, FJU3), à effectif important (EFF3, EFF5), à croissance forte (CRC4), avec une clientèle assez largement répartie (REP2, REP4) et des dirigeants plutôt participatifs (PPD4), telles les entreprises 14 et 27, et, d'autre part, des entreprises individuelles (FJU1) aux caractéristiques opposées (CRC2 et CRC3, REP1, PPD1 et PPD2), comme les entreprises 08 et 28.

\section{Le troisième et le quatrième axes factoriels}

Le troisième axe est plus difficile à interpréter, les variables les plus contributives (EFF, SEC, FJU, AGE) étant essentiellement descriptives, deux seules (TRA, IND) étant stratégiques avec, en outre, des modalités dispersées. Les entreprises caractéristiques de l'axe 3 sont, pour la partie positive, les entreprises 08 et 26, des entreprises du bâtiment à effectif fort (EFF4) et, pour 
la partie négative, l'entreprise 01 , une entreprise de réparation à effectif encore plus important (EFF5).

Le quatrième axe est plus intéressant, avec des variables contributives (LEA, TX, REN, DIS, CRC) bien regroupées aux extrémités de l'axe. Sur la partie positive de l'axe 4, apparaissent des entreprises à forte croissance du chiffre d'affaires (CRC4), à bonne rentabilité actuelle (REN4) et qui recherchent une croissance soutenue dans l'avenir (TX3), telles les entreprises 04 et 14. Sur la partie négative de l'axe, figurent des entreprises moins performantes dans ces trois domaines (CRC1, REN3, TX1), comme les entreprises 16 et 24.

\section{Le cinquième et le sixième axes factoriels}

Le cinquième axe factoriel est constitué essentiellement par deux entreprises aux caractéristiques opposées ; l'une, l'entreprise 23, a un jeune dirigeant (AGD1), a embauché ces deux dernières années (VEF4), a un fort taux de croissance actuel (CRC5) et souhaité (TX5), et son dirigeant accorde beaucoup d'importance à la distribution (DIS5) et à une planification très élaborée (PLA5) ; l'autre, l'entreprise 16, a un dirigeant plus âgé (AGD4), des performances plus limitées (VEF2, CRC1, TX1) et pas de planification (PLA1).

Le sixième axe est constitué essentiellement par deux entreprises sur la partie négative de l'axe et, dans une moindre mesure, par une entreprise sur la partie positive. Les deux entreprises sur la partie négative, la 21 et la 28, ont une croissance modérée (CRC2), mais une forte croissance souhaitée (TX4, TX5), une forte complexité technologique (TEC4) et un dirigeant proactif vis-à-vis de l'environnement (AEN4). Sur la partie positive de l'axe, l'entreprise 09 a une croissance actuelle (CRC1) et une croissance souhaitée (TX1) faibles, une technologie simple (TEC2) et un dirigeant moins agressif envers la concurrence (AEN3).

L'ACM fait ainsi apparaître, axe par axe, des regroupements d'entreprises qui suggèrent divers stades ou diverses caractéristiques de l'émergence. L'établissement d'une typologie de ces entreprises, basée sur les modalités les plus contributives de chaque axe, permet de préciser ces indications. C'est l'objet de la $\mathrm{CAH}$, résumé et synthèse de l'ACM.

\subsubsection{La classification ascendante hiérarchique}

Plusieurs classifications ont été essayées sur l'ACM précédente. Une première classification a été effectuée sur les 28 entreprises, mais, comme on pouvait s'y attendre, la première classe est composée des deux seules entreprises " originales » : la 06 et la 07 ; aussi ces deux entreprises ont-elles été mises en 
éléments supplémentaires. Comme les variables et les modalités sont très nombreuses, afin de ne pas perdre la richesse de l'information, la CAH a été réalisée sur 20 facteurs puis sur 25 facteurs.

Nous commenterons la CAH sur 25 facteurs laquelle paraît plus exhaustive, en recherchant la signification des changements de classe. Afin de mettre en évidence les facteurs caractéristiques de chaque classe, nous avons établi un tableau récapitulatif des 28 entreprises avec les variables les plus contributives des axes de l'ACM (voir tableau 5, page suivante).

Il apparaît ainsi cinq classes d'entreprises, plus une classe exceptionnelle composée des deux entreprises « originales » que nous avons appelées, en fonction de leurs caractéristiques :

- les « valeurs sûres »;

- les « sous-traitantes»;

- les « stratégiques»;

- les « adaptables»;

- les « jeunes loups ».

La classe $1:$ les « valeurs sûres »

Il s'agit d'entreprises du bâtiment $(08,13,26)$ et d'une entreprise de services (28) déjà anciennes (plus de 12 ans, sauf une), à effectif de 16 à 20 personnes (sauf une plus petite), ayant, pour deux d'entre elles, embauché de 1 à 3 personnes ces deux dernières années. Elles bénéficient d'une croissance du chiffre d'affaires satisfaisante (de 11 à $20 \%$ en général) et d'une bonne rentabilité : leur clientèle est essentiellement locale (une seule a une clientèle régionale) et elles ont un ou quelques produits leaders. Elles sont peu ou pas sous-traitantes et la complexité de leur outil de production est moyenne, voire assez forte. Leur degré d'informatisation est assez important (sauf pour une). Leurs dirigeants sont peu participatifs dans la prise de décision, mais très proactifs vis-à-vis de l'environnement et coopèrent volontiers avec d'autres entreprises locales; ils accordent beaucoup d'importance à leur part de marché et au système de distribution ; toutes envisagent une expansion de leur marché actuel et des investissements dans les deux ans à venir, avec la possibilité de fabriquer et de vendre des produits nouveaux. La composition de cette classe reste identique dans les deux CAH.

On peut considérer que les entreprises les plus anciennes $(08,26$, 28) passent actuellement d'un stade de réussite-désengagement à un stade de réussite-expansion; avec trois ou quatre DAS qu'elles satisfont avec une gamme assez étendue de produits et une bonne insertion dans les réseaux locaux, elles font figure de leader dans le marché local,mais en raison des difficultés actuelles du bâtiment, elles cherchent à s'implanter dans un marché plus vaste. 
TABleaU 5

Variables les plus contributives des 28 entreprises regroupées en 5 classes

\begin{tabular}{|c|c|c|c|c|c|c|c|c|c|c|c|c|c|c|c|c|c|c|c|c|c|c|c|c|c|}
\hline \multirow{2}{*}{ Nom de la classe } & \multirow{2}{*}{$\begin{array}{l}\text { Varlables } \\
\text { Eatreprises }\end{array}$} & \multicolumn{4}{|c|}{ Caractires } & \multicolumn{2}{|c|}{ Effeetif } & \multicolumn{2}{|c|}{ Performance } & \multicolumn{4}{|c|}{ Perspectl ves } & \multicolumn{2}{|c|}{ Production } & \multicolumn{2}{|c|}{ Marchts } & \multicolumn{2}{|c|}{ Dirigeant } & \multicolumn{4}{|c|}{$\begin{array}{l}\text { Perception } \\
\text { strategique }\end{array}$} & \multicolumn{2}{|c|}{$\begin{array}{c}\text { Entreprise } \\
\text { Environne ment }\end{array}$} \\
\hline & & SEC & ACE & ACD & FuU & EFF & Vef & CRC & REN & DX & NEW & PER & $\mathbf{N P}$ & TRA & TEC & LEA & REP & PPD & AEN & MAR & DIS & $\mathbf{F L} \mathbf{X}$ & PLA & $\mathbf{N} \mathbf{F}$ & ENT \\
\hline Les valeurs sores & $\begin{array}{c}8 \\
13 \\
26 \\
28\end{array}$ & $\begin{array}{l}\text { BATI } \\
\text { BATI } \\
\text { BATI } \\
\text { SERV }\end{array}$ & $\begin{array}{l}4 \\
2 \\
4 \\
4\end{array}$ & $\begin{array}{l}3 \\
2 \\
3 \\
3\end{array}$ & $\begin{array}{l}1 \\
2 \\
2 \\
1\end{array}$ & $\begin{array}{l}4 \\
4 \\
4 \\
2\end{array}$ & $\begin{array}{l}2 \\
3 \\
2 \\
3\end{array}$ & $\begin{array}{l}3 \\
3 \\
3 \\
2\end{array}$ & $\begin{array}{l}3 \\
4 \\
4 \\
4\end{array}$ & $\begin{array}{l}1 \\
1 \\
3 \\
4\end{array}$ & $\begin{array}{l}3 \\
3 \\
3 \\
3\end{array}$ & $\begin{array}{l}2 \\
2 \\
2 \\
2\end{array}$ & $\begin{array}{l}3 \\
3 \\
3 \\
3\end{array}$ & $\begin{array}{l}1 \\
2 \\
2 \\
1\end{array}$ & $\begin{array}{l}3 \\
4 \\
3 \\
4\end{array}$ & $\begin{array}{l}2 \\
3 \\
2 \\
2\end{array}$ & $\begin{array}{l}1 \\
2 \\
1 \\
1\end{array}$ & $\begin{array}{l}2 \\
2 \\
2 \\
1\end{array}$ & $\begin{array}{l}4 \\
5 \\
5 \\
4\end{array}$ & $\begin{array}{l}4 \\
4 \\
4 \\
4\end{array}$ & $\begin{array}{l}4 \\
4 \\
4 \\
3\end{array}$ & $\begin{array}{l}1 \\
5 \\
5 \\
5\end{array}$ & $\begin{array}{l}3 \\
2 \\
2 \\
1\end{array}$ & $\begin{array}{l}3 \\
4 \\
3 \\
1\end{array}$ & $\begin{array}{l}2 \\
2 \\
2 \\
1\end{array}$ \\
\hline Les sous-traltents & $\begin{array}{l}2 \\
4 \\
11 \\
12 \\
15 \\
20 \\
21 \\
27\end{array}$ & $\begin{array}{l}\text { PROD } \\
\text { PROD } \\
\text { PROD } \\
\text { PROD } \\
\text { REPA } \\
\text { PROD } \\
\text { PROD } \\
\text { PROD }\end{array}$ & $\begin{array}{l}3 \\
2 \\
4 \\
5 \\
3 \\
4 \\
2 \\
2\end{array}$ & $\begin{array}{l}3 \\
2 \\
3 \\
3 \\
3 \\
2 \\
2 \\
2\end{array}$ & $\begin{array}{l}2 \\
2 \\
2 \\
1 \\
1 \\
2 \\
2 \\
2\end{array}$ & $\begin{array}{l}3 \\
2 \\
2 \\
3 \\
1 \\
2 \\
5 \\
3\end{array}$ & $\begin{array}{l}2 \\
4 \\
3 \\
3 \\
2 \\
2 \\
5 \\
1\end{array}$ & $\begin{array}{l}1 \\
4 \\
2 \\
3 \\
2 \\
2 \\
2 \\
4\end{array}$ & $\begin{array}{l}4 \\
4 \\
4 \\
2 \\
4 \\
4 \\
1 \\
2\end{array}$ & $\begin{array}{l}3 \\
3 \\
1 \\
2 \\
2 \\
3 \\
2 \\
1\end{array}$ & $\begin{array}{l}3 \\
2 \\
1 \\
3 \\
1 \\
3 \\
3 \\
3\end{array}$ & $\begin{array}{l}2 \\
2 \\
1 \\
2 \\
2 \\
2 \\
2 \\
2\end{array}$ & $\begin{array}{l}3 \\
2 \\
2 \\
2 \\
1 \\
2 \\
3 \\
3\end{array}$ & $\begin{array}{l}5 \\
2 \\
3 \\
2 \\
4 \\
5 \\
4 \\
5\end{array}$ & $\begin{array}{l}2 \\
2 \\
2 \\
1 \\
1 \\
4 \\
4 \\
3\end{array}$ & $\begin{array}{l}2 \\
1 \\
2 \\
3 \\
2 \\
1 \\
2 \\
1\end{array}$ & $\begin{array}{l}2 \\
3 \\
2 \\
4 \\
3 \\
2 \\
4 \\
2\end{array}$ & $\begin{array}{l}2 \\
1 \\
3 \\
3 \\
2 \\
2 \\
2 \\
4\end{array}$ & $\begin{array}{l}3 \\
3 \\
4 \\
4 \\
4 \\
4 \\
4 \\
4\end{array}$ & $\begin{array}{l}5 \\
4 \\
4 \\
3 \\
2 \\
3 \\
4 \\
3\end{array}$ & $\begin{array}{l}4 \\
2 \\
2 \\
4 \\
3 \\
1 \\
4 \\
4\end{array}$ & $\begin{array}{l}5 \\
2 \\
4 \\
5 \\
4 \\
3 \\
4 \\
5\end{array}$ & $\begin{array}{l}4 \\
1 \\
1 \\
1 \\
1 \\
1 \\
3 \\
1\end{array}$ & $\begin{array}{l}1 \\
2 \\
2 \\
2 \\
1 \\
1 \\
2 \\
2\end{array}$ & $\begin{array}{l}1 \\
1 \\
1 \\
1 \\
1 \\
1 \\
1 \\
1\end{array}$ \\
\hline Les stratteglques & $\begin{array}{l}14 \\
23 \\
24\end{array}$ & $\begin{array}{l}\text { SERV } \\
\text { PROD } \\
\text { REPA }\end{array}$ & $\begin{array}{l}3 \\
1 \\
1\end{array}$ & $\begin{array}{l}2 \\
1 \\
2\end{array}$ & $\begin{array}{l}3 \\
2 \\
2\end{array}$ & $\begin{array}{l}5 \\
2 \\
2\end{array}$ & $\begin{array}{l}5 \\
4 \\
1\end{array}$ & $\begin{array}{l}4 \\
5 \\
1\end{array}$ & $\begin{array}{l}4 \\
3 \\
3\end{array}$ & $\begin{array}{l}3 \\
5 \\
1\end{array}$ & $\begin{array}{l}3 \\
3 \\
3\end{array}$ & $\begin{array}{l}2 \\
2 \\
2\end{array}$ & $\begin{array}{l}3 \\
3 \\
3\end{array}$ & $\begin{array}{l}1 \\
3 \\
1\end{array}$ & $\begin{array}{l}1 \\
3 \\
3\end{array}$ & $\begin{array}{l}3 \\
2 \\
4\end{array}$ & $\begin{array}{l}5 \\
4 \\
2\end{array}$ & $\begin{array}{l}4 \\
2 \\
3\end{array}$ & $\begin{array}{l}5 \\
4 \\
4\end{array}$ & $\begin{array}{l}5 \\
4 \\
5\end{array}$ & $\begin{array}{l}4 \\
5 \\
5\end{array}$ & $\begin{array}{l}5 \\
5 \\
5\end{array}$ & $\begin{array}{l}2 \\
5 \\
3\end{array}$ & $\begin{array}{l}2 \\
3 \\
2\end{array}$ & $\begin{array}{l}2 \\
1 \\
1\end{array}$ \\
\hline Les adaptables & $\begin{array}{l}10 \\
18 \\
19 \\
22 \\
25\end{array}$ & \begin{tabular}{|l} 
BATI \\
BATI \\
ALM \\
BATI \\
ALM
\end{tabular} & $\begin{array}{l}3 \\
3 \\
3 \\
1 \\
2\end{array}$ & $\begin{array}{l}2 \\
2 \\
3 \\
2 \\
2\end{array}$ & $\begin{array}{l}1 \\
1 \\
1 \\
2 \\
1\end{array}$ & $\begin{array}{l}2 \\
2 \\
3 \\
1 \\
2\end{array}$ & $\begin{array}{l}2 \\
3 \\
3 \\
2 \\
2\end{array}$ & $\begin{array}{l}3 \\
5 \\
3 \\
5 \\
2\end{array}$ & $\begin{array}{l}4 \\
4 \\
4 \\
2\end{array}$ & $\begin{array}{l}1 \\
5 \\
3 \\
5 \\
2\end{array}$ & $\begin{array}{l}1 \\
1 \\
3 \\
3 \\
1\end{array}$ & $\begin{array}{l}2 \\
2 \\
2 \\
2 \\
1\end{array}$ & $\begin{array}{l}1 \\
1 \\
3 \\
3 \\
1\end{array}$ & $\begin{array}{l}2 \\
1 \\
1 \\
1 \\
1\end{array}$ & $\begin{array}{l}3 \\
1 \\
2 \\
5 \\
1\end{array}$ & $\begin{array}{l}2 \\
2 \\
2 \\
2 \\
2\end{array}$ & $\begin{array}{l}2 \\
1 \\
2 \\
2 \\
1\end{array}$ & $\begin{array}{l}1 \\
2 \\
1 \\
1 \\
1\end{array}$ & $\begin{array}{l}4 \\
3 \\
4 \\
4 \\
3\end{array}$ & $\begin{array}{l}4 \\
3 \\
4 \\
4 \\
2\end{array}$ & $\begin{array}{l}2 \\
1 \\
2 \\
3 \\
4\end{array}$ & $\begin{array}{l}5 \\
4 \\
4 \\
5 \\
4\end{array}$ & $\begin{array}{l}2 \\
2 \\
1 \\
1 \\
1\end{array}$ & $\begin{array}{l}3 \\
1 \\
1 \\
1 \\
1\end{array}$ & $\begin{array}{l}1 \\
1 \\
1 \\
2 \\
1\end{array}$ \\
\hline Les arrivtes & $\begin{array}{c}1 \\
3 \\
5 \\
9 \\
16 \\
17\end{array}$ & $\begin{array}{l}\text { REPA } \\
\text { REPA } \\
\text { ALM } \\
\text { ALM } \\
\text { BATT } \\
\text { PROD }\end{array}$ & $\begin{array}{l}3 \\
3 \\
5 \\
3 \\
4 \\
5\end{array}$ & $\begin{array}{l}4 \\
4 \\
4 \\
4 \\
4\end{array}$ & $\begin{array}{l}3 \\
2 \\
1 \\
1 \\
2 \\
1\end{array}$ & $\begin{array}{l}5 \\
3 \\
3 \\
2 \\
1 \\
1\end{array}$ & $\begin{array}{l}3 \\
2 \\
3 \\
3 \\
2 \\
3\end{array}$ & $\begin{array}{l}2 \\
5 \\
2 \\
1 \\
1 \\
3\end{array}$ & $\begin{array}{l}4 \\
2 \\
4 \\
3 \\
3 \\
3\end{array}$ & $\begin{array}{l}2 \\
1 \\
2 \\
1 \\
1 \\
1\end{array}$ & $\begin{array}{l}3 \\
3 \\
3 \\
3 \\
1 \\
2\end{array}$ & $\begin{array}{l}1 \\
2 \\
2 \\
2 \\
1 \\
2\end{array}$ & $\begin{array}{l}1 \\
2 \\
3 \\
3 \\
1 \\
1\end{array}$ & $\begin{array}{l}1 \\
1 \\
1 \\
1 \\
1 \\
1\end{array}$ & $\begin{array}{l}3 \\
3 \\
1 \\
2 \\
1 \\
5\end{array}$ & $\begin{array}{l}3 \\
3 \\
3 \\
3 \\
2 \\
2\end{array}$ & $\begin{array}{l}2 \\
1 \\
1 \\
3 \\
2 \\
1\end{array}$ & $\begin{array}{l}3 \\
2 \\
3 \\
2 \\
1 \\
1\end{array}$ & $\begin{array}{l}5 \\
5 \\
4 \\
3 \\
3 \\
5\end{array}$ & $\begin{array}{l}5 \\
4 \\
4 \\
4 \\
2 \\
4\end{array}$ & $\begin{array}{l}5 \\
4 \\
2 \\
4 \\
4 \\
4\end{array}$ & $\begin{array}{l}4 \\
4 \\
5 \\
4 \\
3 \\
4\end{array}$ & $\begin{array}{l}1 \\
3 \\
2 \\
1 \\
1 \\
1\end{array}$ & $\begin{array}{l}4 \\
2 \\
1 \\
3 \\
1 \\
3\end{array}$ & $\begin{array}{l}1 \\
1 \\
2 \\
2 \\
1 \\
1\end{array}$ \\
\hline Les Jeunes loups & 9 & $\begin{array}{l}\text { BATI } \\
\text { BATI }\end{array}$ & $\begin{array}{l}1 \\
1\end{array}$ & 1 & $\begin{array}{l}2 \\
2\end{array}$ & $\begin{array}{l}3 \\
4\end{array}$ & $\begin{array}{l}2 \\
4\end{array}$ & $\begin{array}{l}5 \\
4\end{array}$ & 1 & $\begin{array}{l}3 \\
3\end{array}$ & $\begin{array}{l}3 \\
3\end{array}$ & 2 & $\begin{array}{l}3 \\
1\end{array}$ & 2 & $\begin{array}{l}3 \\
3\end{array}$ & 2 & $\begin{array}{l}2 \\
4\end{array}$ & $\begin{array}{l}3 \\
3\end{array}$ & $\begin{array}{l}5 \\
4\end{array}$ & $\begin{array}{l}5 \\
5\end{array}$ & 2 & $\begin{array}{l}2 \\
2\end{array}$ & $\begin{array}{l}1 \\
1\end{array}$ & $\begin{array}{l}3 \\
3\end{array}$ & $\begin{array}{l}1 \\
1\end{array}$ \\
\hline
\end{tabular}


L'entreprise 13, plus jeune et la plus avancée du point de vue technologique et commercial, paraît en phase continue de réussite-expansion. La bonne qualité d'ensemble de ces entreprises amène à les considérer comme des « valeurs sûres ».

\section{La classe 2 : les « sous-traitantes 》}

Cette classe regroupe huit entreprises de production $(02,04,11,12,15,20,21$, 27) d'âges divers, largement sous-traitantes, avec une clientèle plus étendue que celle de la classe précédente (de la région à la France entière), mais de taille souvent plus petite, peu ou pas informatisées et aux performances variables en matière de croissance et de rentabilité. Leurs dirigeants sont assez proactifs face à l'environnement, font preuve d'une certaine perception stratégique, mais à la différence dos précédents, ne coopèrent pas avec d'autres entreprises locales; ils envisagent en général une expansion de leur marché actuel, mais restent parfois incertains quant à leurs investissements futurs.

C'est dans cette classe que le changement de composition est le plus important : elle varie de 8 entreprises dans la CAH 25 facteurs à 6 entreprises dans la CAH 20 facteurs. L'entreprise 21 passe ainsi dans les « stratégiques » en raison de la perception stratégique du dirigeant, et les entreprises 02 et 15 passent dans les « arrivées », vraisemblablement en raison de leur âge moyen et de leur croissance limitée. L'entreprise 16 passe des « arrivées » aux « soustraitantes », bien qu'elle ne soit pas sous-traitante (!) et soit située dans les valeurs les plus faibles de la plupart des variables, sauf en ce qui concerne la rentabilité satisfaisante et l'âge important. Les entreprises stables de cette classe sont les $04,11,12,20,21$ et 27 . L'entreprise 27 , fortement sous-traitante, avec une compétence technologique certaine et des possibilités de produits nouveaux et performants, est au stade de la réussite-expansion, à la recherche d'un développement autonome basé sur un savoir-faire acquis dans la sous-traitance. Les entreprises 20 et 21 sont assez semblables, mais la 21 est pénalisée par une croissance modérée et une faible rentabilité et la 20 , plus âgée, est incertaine quant à ses investissements futurs malgré une certaine ambition en matière de croissance pour l'avenir. Les autres entreprises, plus faiblement sous-traitantes, paraissent se situer dans un stade de réussite-désengagement avec des performances et des ambitions limitées et des perspectives d'investissement incertaines, à l'exception peut-être de la 04 qui réalise une forte croissance avec une bonne rentabilité.

\section{La classe 3 : les « stratégiques»}

Dans cette classe figurent les entreprises $14,23,24$, la première plus âgée et de plus grande taille, les deux autres jeunes et de très petite taille, appartenant à des secteurs différents. Toutes ont une forme sociétaire, une rentabilité satisfaisante, 
une clientèle répartie dans la région ou la France et, pour la plus grande, dans l'Europe avec plusieurs produits leaders. Leurs dirigeants, jeunes, sont très proactifs vis-à-vis de l'environnement et manifestent une perception stratégique affirmée, aussi bien en matière de marketing que de flexibilité : parmi tous les dirigeants, ce sont eux qui accordent le plus d'importance à la planification ; ils envisagent tous un développement de leur marché, avec des produits nouveaux et des investissements dans un proche avenir.

Ces trois entreprises restent dans la même classe avec les deux CAH. Les entreprises 23 et 24 , à la fin du stade de démarrage, disposent des moyens nécessaires pour s'engager dans un stade de réussite-expansion, l'entreprise 24 étant cependant pénalisée par une croissance actuelle plus faible, mais avantagée par plus de produits leaders que l'entreprise 23. L'entreprise 14, en pleine croissance, a atteint le stade de réussite-expansion. Le point commun de ces entreprises qui est en même temps leur atout majeur, c'est la capacité stratégique de leur dirigeant.

\section{La classe 4 : les « adaptables »}

Cette classe regroupe des entreprises du bâtiment $(10,18,22)$ et des entreprises du secteur alimentaire (19 et 25), d'âge moyen et d'effectif limité, à bonne rentabilité dans l'ensemble et qui ont procédé à quelques embauches ces dernières années. Leur clientèle est locale ou régionale et elles ont toutes un produit leader. Leurs dirigeants sont peu participatifs et coopèrent peu avec les autres entreprises locales, mais ils sont plutôt proactifs et font preuve d'une certaine perception stratégique. Ils sont plutôt ambitieux en matière de croissance future et prévoient une extension de leur marché, mais ils n'ont pas tous la possibilité de proposer des produits nouveaux et, pour plus de la moitié, n'envisagent pas d'investissement dans un proche avenir.

La composition de cette classe est la même dans les deux CAH. Une seule de ces entreprises, l'entreprise 22 est au stade de démarrage, avec un dirigeant et des moyens (produits nouveaux, technologie, investissements futurs) lui permettant d'espérer une réussite-expansion. Les autres sont vraisemblablement en réussite-désengagement, sauf l'entreprise 19 qui, avec des perspectives d'investissements et des produits nouveaux, est plutôt en réussite-expansion. Dans l'ensemble, ces entreprises paraissent capables de s'adapter à l'évolution de leur environnement.

\section{La classe 5 : les « arrivées 》}

Cette classe est composée des entreprises $01,03,05,09,16,17$, appartenant à des secteurs variés, d'âge plus important que celles de la classe précédente, mais 
assez semblables dans leur ensemble. Les différences les plus notables sont l'âge des dirigeants (les plus âgés de l'échantillon, de 50 à 59 ans pour la plupart), un nombre de produits leaders plus important, une plus grande capacité à fabriquer des produits nouveaux, mais des ambitions plus limitées en matière de croissance future.

La composition de la classe 5 varie suivant les $\mathrm{CAH}$; dans la $\mathrm{CAH}$ 20 facteurs, elle comprenait 7 entreprises avec l'entreprise 02 et l'entreprise 15 à la place de l'entreprise 16. Les entreprises stables de la classe 5 sont les 01 , 03, 05, 09 et 17 ; elles sont arrivées à un certain niveau de réussite, réussitedésengagement, voire réussite-expansion pour la plus grande (la 01) et elles s'y sont maintenues ; certaines (la 05 et la 09) ont des perspectives d'investissement. Dans l'ensemble, ces entreprises donnent l'impression d'attendre un successeur ambitieux pour prendre un nouveau départ.

\section{La classe exceptionnelle : les « jeunes loups »}

Les entreprises 06 et 07 à elles seules ne constituent pas une véritable classe, mais la convergence de leurs caractéristiques les a fait ressortir de l'ensemble étudié. Il s'agit d'entreprises du bâtiment, de taille assez importante, jeunes, moyennement informatisées, à forte croissance avec un produit leader et une clientèle allant de la région à la France entière, mais à faible rentabilité actuelle. Leurs dirigeants jeunes, paraissent participatifs et très proactifs, mais peu coopératifs avec les entreprises locales ; ils sont très attentifs à leur marché, mais ne planifient pas encore leur développement.

Ces deux entreprises, au stade du démarrage, peuvent s'engager dans une réussite-expansion, avec des produits nouveaux, à condition de mettre en œuvre une véritable planification stratégique.

\section{Conclusion}

La méthode du diagnostic d'émergence proposée donne des résultats intéressants, comme le montre l'exemple de l'échantillon étudié.

L'ACM, en faisant ressortir les variables les plus significatives, met en évidence les éléments déclencheurs de l'émergence qui sont :

- Le comportement du dirigeant, notamment son attitude proactive face à l'environnement, et sa perception stratégique en matière de marché, de flexibilité et de planification. 
- Le caractère offensif de sa stratégie et ses composants, en particulier la technologie mise en œuvre, le nombre de produits leaders et l'étendue de sa clientèle, le degré d'informatisation et la coopération avec d'autres entreprises.

L'ACM fait aussi apparaître les entreprises qui émergent le plus rapidement, celles en phase de démarrage favorable comme l'entreprise 23, et celles en phase de réussite-expansion comme les entreprises 13, 14 (qui ressort trois fois dans l'ACM) et 27.

La CAH détermine des classes d'entreprises dont certaines paraissent plus aptes à l'émergence, plus particulièrement les "stratégiques » en raison de la capacité stratégique de leurs dirigeants, et les « valeurs sûres » en raison de leurs bonnes performances d'ensemble. Mais l'intérêt de cette classification est de montrer que l'émergence ne s'effectue pas forcément selon un processus continu. Si l'on peut considérer que les « stratégiques » se développent assez régulièrement, les « valeurs sûres », les « adaptables » et les " arrivées » font apparaître des pauses qui peuvent être longues comme dans le stade de réussitedésengagement, ou même des retours en arrière, de l'expansion au désengagement. La reprise de l'expansion reste cependant toujours possible par le jeu de nouveaux ćléments déclencheurs (changement de dirigeant, saisie de nouvelles opportunités offertes par la turbulence des marchés...).

La mise en évidence des différentes classes d'entreprises permet à l'organisme public intéressé par cette ćtude de mieux définir des programmes d'accompagnement. Pour les "valeurs sûres » et les "stratégiques » qui ont une vision d'ensemble de leur développement et des moyens à mettre en œuvre, les mesures ne peuvent être que ponctuelles, à l'occasion de besoins spécifiques. Pour les « arrivées », il s'agit surtout d'une aide à la transmission d'entreprise, lorsque se posera prochainement le problème de la succession du dirigeant. Pour les " sous-traitants », il paraît utile de les aider à diversifier leur activité et leur clientèle, à partir d'un savoir-faire évident. Pour les « adaptables », une assistance se révèle nécessaire pour mieux définir leurs segments de marché futurs, des produits nouveaux avec un positionnement adéquat, ainsi que les investissements nécessaires. Enfin, pour les « jeunes loups », l'aide essentielle porte sur la planification du développement.

Il serait intéressant de tester l'efficacité de ce diagnostic d'émergence en l'appliquant à un échantillon plus étendu et plus précisément à des entreprises en démarrage, afin de rechercher celles qui s'engageront directement dans une phase de réussite-expansion. 


\section{Bibliographie}

AltmaN, E.I. (1968), « Financial ratios, discriminant analysis and the prediction of corporate bankruptcy », The Journal of Finance, vol. 28, septembre, p. 589-689.

Altman, E.I. (1983), «Why businesses fail », Journal of Business Strategy, vol. 3, $\mathrm{n}^{\circ}$ 4. «Exploring the road to bankruptcy », Journal of Business Strategy, vol. 4, $\mathrm{n}^{\circ} 2$.

ARCHer, A. (1990), "Un diagnostic de pré-démarrage pour les PME et PE », Revue Internationale P.M.E., vol. 3, $\mathrm{n}^{\circ}$ s 3-4, p. 389-409.

ARgenti, J. (1976), Corporate Collapse, New York, McGraw-Hill.

ARGENTI, J. (1977), “Company failure. Long range prediction not enough», Accountancy, août.

BEAVER, W.H. (1966), «Financial ratios as predictor of failure. Empirical research », Journal of Accounting Research, supplément $n^{\circ} 4$, p. 71-111.

Belletante, B. (1991), « Pour une approche des spécificités financières de la PME au travers du concept de territoire financier », Revue Internationale P.M.E., vol. 4, $n^{\circ} 1$, p. 49-79.

BERRYMAN, J. (1983), «Small business failure and bankruptcy : a survey of the literature », European Small Business Journal, vol. 1, $\mathrm{n}^{\circ} 4$, p. 41-59.

CAPIEZ, A. (1988), «Conditions et modalités d'émergence des TPE et PE : l'exemple du Maine-et-Loire (France) », Revue Internationale P.M.E., vol. 1, $\mathrm{n}^{\circ}$ 2, p. 128-155.

Carland, J., F. Hoy, W. Boulton et J.A. Carland (1984), « Differenciating entrepreneurs from small business owners : a conceptualization », Academy of Management Review, vol. 9, p. 354-359.

Churchill, N. et V. Lewis (1983), "Les cinq stades d'évolution d'une PME », Harvard-L'expansion, automne, p. 51-63.

CoOper, A. et C. DUNKElberg (1982), «Entrepreneurial typologies : an empirical study », dans Frontiers of Entrepreneurship Research, Babson College.

COOPER, A., W. Dulkenberg et C. Woo (1988), "Survival and failure : a longitudinal study ", dans Frontiers of Entrepreneurship Research, Babson College, p. 225-237.

DeEks, J. (1973), « The small firm. Asset or liability? ", Journal of Management Studies, vol. $10, \mathrm{n}^{\circ} 1$, p. 25-47.

DUBINI, P. (1989), «The evaluation of new ventures and entrepreneurs : methodological considerations », in The Evaluation of Entrepreneurial Projects and Profiles, EGEA, Milan, p. 103-122. 
FILEY, A. et R. ALDAG (1978), « Characteristics and measurement of an organizational typology 》, Academy of Management Journal, vol. 21, n 4, p. 578-591.

FOURCADE, C. (1986), "Le démarrage des entreprises : une comparaison internationale », dans La PME dans un monde en mutation, Montréal, Presses de l'Université du Québec, p. 253-277.

GeLINIRR, O. et A. GAULTIER (1974), «L'avenir des entreprises personnelles et familiales », Éditions Hommes et Techniques, Puteaux.

GREINER, L. (1972), «Evolution and revolution of organizations growth », Harvard Business Review, vol. 50, $\mathrm{n}^{\circ}$ 4, p. 37-46.

HoldER, M., J. LOEB et G. PORTIER (1984), Le score de l'entreprise, Nouvelles éditions fiduciaires, Paris.

JULIEN, P.A. (1990), « Vers une typologie multicritère des PME », Revue Internationale P.M.E., vol. 3, nos 3-4, p. 411-425.

Julien, P.A. et J. Chicha (1982), "Vers une typologie multicritère des PME manufacturières », Cahiers de recherche du GREPME, $n^{\circ}$ 82-06.

Julien, P.A. et M. MARChESNAY (1988), La petite entreprise, Paris, Vuibert, p. 70-73.

KoENING, G. (1985), « Entreprises en difficultés : des symptômes aux remèdes », Revue Française de Gestion, janvier - février. 1985, p. 84-92.

LIVIAN, Y. et S. MARION (1991), « De l'évaluation des projets de création d'entreprises au pronostic de succès », Revue Internationale PME, vol. 4, n 1, p. 107-129.

LILES, P. (1974), New Business Ventures and the Entrepreneur, Homewood, R.D. Irwin.

MacMIllan, I., R. Siegel et P. Subra Narashima (1985), "Criteria used by venture capitalists to evaluate new venture proposals ", Journal of Business Venturing, vol. $1, \mathrm{n}^{\circ} 1, \mathrm{p} .123-138$.

MALECoT, J.F. (1981), «Les défaillances : un essai d'explication », Revue Française de gestion, septembre - octobre 1981, p. 10-18.

MaLeCot, J.F. (1989), « Gestion financière de l'entreprise en difficultés 》, dans Encyclopédie de Gestion, Paris, Économica.

MARCHESNAY, M. (1985), « L'artisanat dans un monde en mutation », Enseignement et Gestion, $\mathrm{n}^{\circ} 35$.

Marchesnay, M. (1986), La stratégie, Paris, Chotard.

MASSACRIER, G. et G. RIGAUD (1984), « Le démarrage d'activités nouvelles : aléas et processus », Revue Française de Gestion, mars - mai, p. 5-18.

MILlER, D. (1977), « Common-Syndrome of Business Failure », Business-Horizon, T. 20, no. 6. 
NeISWANDER, D. et J. DrollingeR (1986), « Origins of successful start-up ventures », dans Frontiers of Entrepreneurship Research, Babson College, p. 328-334.

Peterson, R.A., G. Kozmetsky et Ridgway (1983), " Perceived causes of small business failures : a research note », American Journal of Small Business, vol. $8, \mathrm{n}^{\circ} 1$.

RAMUS, V. (1989), « A longitudinal study of new business projects : first results about the early development of new ventures ", dans P. Dubini, The Evaluation of Entrepreneurial Projects and Profiles, EGEA, Milan, p. 73-92.

RioAud, G. (1987), « Le démarrage d'activités nouvelles : typologie des risques et financement des projets », Banque, $\mathrm{n}^{\circ}$ 504, p. 244-260.

STUART, R. et P. ABETTI (1987), « Start-up ventures : toward the prediction of initial success », Journal of Business Venturing, vol. 2, $\mathrm{n}^{\circ} 3$, p. 215-230.

STUART, R. et P. ABETTI (1988), « Field study of technical ventures. The impact of entrepreneurial and management experience on early performance ", dans Frontiers of Entrepreneurship Research, Babson College, p. 177-193.

VARGAS, G. (1984), «Les crises de croissance de la PMI-PME », Revue Française de Gestion, janvier - février. 